\title{
The Chebyshev Polynomial of Best Approxi- mation to a Given Function on an Interval
}

\author{
By O. Shisha
}

1. Let $n$ be a positive integer, and let $P_{n}$ denote the set of all polynomials $\sum_{j=0}^{n} a_{j} x^{j}, a_{j}$ real. It is known that for every real, finite set $C$ containing at least $n+1$ points and for every real function $f$ defined on $C$ there exists a unique $q \in P_{n}$ such that, for every $p \in P_{n}$,

$$
\max _{x \in C}|f(x)-q(x)| \leqq \max _{x \in C}|f(x)-p(x)| .
$$

Furthermore, the determination of this $q$ can be carried out by known methods involving arithmetic operations only, and one can even give at the outset an upper bound (perhaps large) for the number of the arithmetic operations necessary. For instance, the determination of $q$ can be viewed as a linear programming problem.

2. Let $f$ be a real function defined and continuous on $[0,1]$. Consider the problem of determining the (unique) $\tilde{p} \in P_{n}$ such that for every $p \in P_{n}$,

$$
\max _{0 \leqq x \leqq 1}|f(x)-\tilde{p}(x)| \leqq \max _{0 \leqq x \leqq 1}|f(x)-p(x)| .
$$

It has been shown [1] that given a positive $\eta$, there is a finite subset $C$ of $[0,1]$ (containing at least $n+1$ points) such that the $q \in P_{n}$ satisfying (1) for every $p \in P_{n}$ is within less than $\eta$ from $\tilde{p}$ throughout $[0,1]$, i.e.,

$$
\max _{0 \leqq x \leqq 1}|\tilde{p}(x)-q(x)|<\eta .
$$

3. Our purpose is to give such a $C$ in a completely closed form, assuming that $f$ satisfies a Lipschitz condition (Theorem 2 ), or has a continuous $(n+1)$ st derivative (Thereom 1). We make use of de la Vallée Poussin's technique [1], but employ also some other results.

4. Theorem 1. Let $n(\geqq 1)$ be an integer, $f$ a real function such that $f^{(n+1)}$ is continuous at each point of $[0,1]$. Let $S_{n+2}$ be an arbitrary $(n+2)$-point subset of $[0,1]$,

$$
\rho_{1}=\min _{\boldsymbol{p} \in \boldsymbol{P}_{n}} \max _{x \in \boldsymbol{S}_{n+2}}|f(x)-p(x)|,
$$

and suppose that $\rho_{1}$ (for which there is an explict formula, see Remark 1 below) is positive. Let $\mu, V, \mu_{1}$ and $M$ be numbers such that throughout $[0,1],|f(x)| \leqq \mu,\left|f^{\prime}(x)\right| \leqq \mu_{1}$, $\left|f^{(n+1)}(x)\right| \leqq M$ and $\frac{1}{2}\left[\max _{0 \leqq x \leqq 1} f(x)-\min _{0 \leqq x \leqq 1} f(x)\right] \leqq V$. Let

$$
\begin{aligned}
c= & {\left[M /\left\{\rho_{1}(n+1) !\right\}\right]^{n}(n !)^{-1} \prod_{\nu=0}^{n-1} 1-\left\{2 \rho_{1} \nu(n+1) ! / M\right\}, } \\
\left.U=(n+2)\left[\prod_{\nu=1}^{n} \nu !\right]^{-1}\left[M /\left\{4 \rho_{1}(n+1) !\right\}\right]_{2}^{n+1}\right) & {\left[\left\{\prod_{\nu=2}^{n+1} \nu^{\nu}\right\}\left\{\prod_{\nu=0}^{n-1} \nu^{\nu}\right\}\left\{\prod_{\nu=1}^{n}(2 \nu-1)^{2 \nu-1}\right\}^{-1}\right]^{1 / 2} . }
\end{aligned}
$$

Received September 7, 1965. 
$V$ and $M$ are positive since $\rho_{1}>0$. Also, $c$ is positive as shown below. Let $\eta$ be an arbitrary positive number. Let $\epsilon$ be a positive number such that $\epsilon V[1+\{U /(1+\epsilon)\}] \leqq \eta / c$ (for instance, let $\epsilon$ be $\left.\eta\{c V(1+U)\}^{-1}\right)$. Let $c_{k}=[1+\cos (k \pi / n)] / 2(k=0,1$, $\cdots, n)$. Let $C$ be an arbitrary finite subset of $[0,1]$ containing $\left\{c_{0}, c_{1}, \cdots, c_{n}\right\}$ and such that the maximal distance $d$ between two consecutive points of $C$ is smaller or equal to $\rho_{1} \epsilon /\left(\mu_{1}+4 \mu n^{2}\right)$. Let $\tilde{p}, q$ be respectively, the elements of $P_{n}$ such that

$$
\begin{aligned}
\rho= & \max _{0 \leqq x \leqq 1}|f(x)-\not{p}(x)|=\min _{\boldsymbol{p} \in \boldsymbol{P}_{\boldsymbol{n}} 0 \leqq x \leqq 1} \max _{0}|f(x)-p(x)|, \\
\max _{x \in C}|f(x)-q(x)|= & \min _{\boldsymbol{p} \in P_{\boldsymbol{n}}} \max _{x \in C}|f(x)-p(x)| .
\end{aligned}
$$

Then $\max _{0 \leqq x \leqq 1}|\tilde{p}(x)-q(x)|<\eta$.

REMARK 1. If $S_{n+2}=\left\{y_{0}, y_{1}, \cdots, y_{n+1}\right\}$, with $y_{0}<y_{1} \cdots<y_{n+1}$, then [1]

$$
\rho_{1}=\left|\sum_{\nu=0}^{n+1}(-1)^{\nu} G_{\nu} f\left(y_{\nu}\right)\right| / \sum_{\nu=0}^{n+1} G_{\nu}
$$

where $G_{\nu}=\prod_{0 \leqq \alpha<\beta \leqq n+1 ; \alpha \neq \nu, \beta \neq \nu 0}\left(y_{\beta}-y_{\alpha}\right)(\nu=0,1, \cdots, n+1)$.

In particular, if $y_{\nu}=\nu /(n+1)(\nu=0,1, \cdots, n+1)$, then from (3) one easily obtains

$$
\rho_{1}=\left(\frac{1}{2}\right)^{n+1}\left|\sum_{\nu=0}^{n+1}(-1)^{\nu}\left(\begin{array}{c}
n+1 \\
\nu
\end{array}\right) f(\nu /(n+1))\right| .
$$

REMARK 2. In connection with the definition of $C$ we note that, as is easily seen, the largest distance between two consecutive $c_{k}$ 's is $\sin [\pi /(2 n)]$ if $n$ is odd, and is $\{\sin (n-1) \pi /(2 n)]\} \sin [\pi /(2 n)]$ if $n$ is even.

5. In the proof of Theorem 1 we s'iall use the following Lemma. Let $0 \leqq x_{0}<x_{1} \cdots<x_{n} \leqq 1(n \geqq 1)$, and let

$$
x_{\nu}-x_{\nu-1}>\delta>0 \quad(\nu=1,2, \cdots, n) .
$$

Let $j$ be an integer, $0 \leqq j \leqq n$. Let $x \in[0,1]$. Then

$$
\prod_{\nu=0, \nu \neq j}^{n}\left|x-x_{\nu}\right| /\left|x_{j}-x_{\nu}\right|<\left[\prod_{\nu=0}^{n-1}(1-\nu \delta)\right] /\left[j !(n-j) ! \delta^{n}\right] .
$$

Proof of the Lemma. (4) clearly implies that

$$
\prod_{\nu=0, \nu \neq j}^{n}\left|x_{j}-x_{\nu}\right|>j !(n-j) ! \delta^{n} .
$$

Therefore, to prove (5) it suffices to show that

$$
\prod_{\nu=0, \nu \neq j}^{n}\left|x-x_{\nu}\right| \leqq \prod_{\nu=0}^{n-1}(1-\nu \delta) .
$$

If $x \geqq x_{n}$, then $\prod_{\nu=0, \nu \neq j}^{n}\left|x-x_{\nu}\right| \leqq \prod_{\nu=0, \nu \neq j}^{n}(1-\nu \delta)$. Similarly, (6) holds if $x \leqq x_{0}$. Assume now $x_{0}<x<x_{n}$. We shall prove

$$
\prod_{\nu=0, \nu \neq j}^{n}\left|x-x_{\nu}\right|<\prod_{\nu=0}^{n-1}(1-\nu \delta) .
$$


(a) Suppose $\frac{1}{2} \leqq x<1$. Let $r(1 \leqq r \leqq n)$ be such that $x_{r-1} \leqq x \leqq x_{r}$. Then evidently $(r-1) \delta<x<1-(n-r) \delta$. For $k=r-1, r, \cdots, n-1$, let

$$
a_{k}=\left[\prod_{\nu=0}^{k} x+(\nu+1-r) \delta\right]\left[\prod_{\nu=k+1-r}^{n-r}(1-\nu \delta-x)\right] .
$$

If $r-1 \leqq k<n-1$, then

$$
a_{k+1} / a_{k}=[x+(k+2-r) \delta] /[1-(k+1-r) \delta-x]>1 .
$$

Consequently, we always have $a_{r-1} \leqq a_{n-1}$. Now

$$
\begin{aligned}
\prod_{\nu=0, \nu \neq j}^{n}\left|x-x_{\nu}\right| \leqq\left[\prod_{\nu=0}^{r-1}(x-\nu \delta)\right]\left[\prod_{\nu=0}^{n-r}(1-\nu \delta-x)\right] /|x-\xi| & =a_{r-1} /|x-\xi| \leqq a_{n-1} /|x-\xi|
\end{aligned}
$$

where $\xi$ is $j \delta$ if $j \leqq r-1$, and is $1-(n-j) \delta$ if $j \geqq r$. In the first case

$$
\begin{aligned}
a_{n-1} /|x-\xi| & =\left[\prod_{\nu=0, \nu \neq r-j-1}^{n-1} x+(\nu+1-r) \delta\right](1-(n-r) \delta-x) \\
& <\left[\prod_{\nu=0, \nu \neq r-j-1}^{n-1} 1-(n-1-\nu) \delta\right](1-(n-1) \delta) \leqq \prod_{\nu=0}^{n-1}(1-\nu \delta)
\end{aligned}
$$

(an "empty" product means 1), which proves (7). In the second case also,

$$
\begin{aligned}
a_{n-1} /|x-\xi| & =\left[\prod_{\nu=0}^{n-1} x+(\nu+1-r) \delta\right](1-(n-r) \delta-x) /(1-(n-j) \delta-x) \\
& \leqq \prod_{\nu=0}^{n-1} x+(\nu+1-r) \delta<\prod_{\nu=0}^{n-1}(1-\nu \delta) .
\end{aligned}
$$

(b) Suppose $0<x<\frac{1}{2}$. Let $x_{\nu}{ }^{\prime}=1-x_{n-\nu}(\nu=0,1, \cdots, n)$. Then $0 \leqq x_{0}{ }^{\prime}$ $<x_{1}{ }^{\prime}<\cdots<x_{n}{ }^{\prime} \leqq 1, x_{\nu}{ }^{\prime}-x_{\nu-1}{ }^{\prime}>\delta>0(\nu=1,2, \cdots, n)$. Now $1-x_{n}{ }^{\prime}<x$ $<1-x_{0}^{\prime}$ and so $x_{0}^{\prime}<1-x<x_{n}{ }^{\prime}, \frac{1}{2}<1-x<1$. Thus, $\prod_{\nu=0, \nu \neq j}^{n}\left|x-x_{\nu}\right|$ $=\prod_{\nu=0, \nu \neq n-j}^{n}\left|x-x_{n-\nu}\right|=\prod_{\nu=0, \nu \neq n-j}^{n}\left|1-x-x_{\nu}{ }^{\prime}\right|<\prod_{\nu=0}^{n-1}(1-\nu \delta)$.

6. Proof of Theorem 1. Let $x_{0}, x_{1}, \cdots, x_{n+1}$ be points of $[0,1]$ such that $0 \leqq x_{0}<x_{1} \cdots<x_{n+1} \leqq 1$ and such that for $j=0,1, \cdots, n+1$,

$$
\rho=\left|f\left(x_{j}\right)-\tilde{p}\left(x_{j}\right)\right|=\min _{\boldsymbol{p} \in \boldsymbol{P}_{n}} \max _{0}\left|f\left(x_{j}\right)-p\left(x_{j}\right)\right| \text {. }
$$

Their existence is well-known [1]. By another well-known theorem [1]

$$
x_{\nu}-x_{\nu-1}>2 \rho(n+1) ! / M \geqq 2 \rho_{1}(n+1) ! / M \quad(\nu=1,2, \cdots, n+1),
$$

and so $c>0$. Consider some arbitrary $x_{k}$. Let $u, v$ be consecutive points of $C$ such that $u \leqq x_{k} \leqq v$. Then

$$
\left|f\left(x_{k}\right)-q\left(x_{k}\right)\right| \leqq\left|f\left(x_{k}\right)-f(u)\right|+\left|q\left(x_{k}\right)-q(u)\right|+|f(u)-q(u)| .
$$

* In the theorem as given by the text [1] it is required that $\left|f^{(n+1)}(x)\right|$ be strictly smaller than $M$ throughout $[0,1]$. But it is clear from the proof there, that it is sufficient to assume merely that $\left|f^{(n+1)}(x)\right| \leqq M$ throughout $[0,1]$. 
Now, clearly $|q(x)|<2 \mu$ throughout $C$, and a fortiori throughout $\left\{c_{0}, c_{1}, \cdots, c_{n}\right\}$. Therefore, by a result of Duffin and Schaeffer [2] refining a previous result of A. Markoff [3], $\left|q^{\prime}(x)\right|<4 \mu n^{2}$ throughout [0,1], and so

$$
\left|f\left(x_{k}\right)-q\left(x_{k}\right)\right|<d\left(\mu_{1}+4 \mu n^{2}\right)+\rho \leqq \epsilon \rho_{1}+\rho \leqq(\epsilon+1) \rho .
$$

Let

$$
\rho^{\prime}=\max _{0 \leqq j \leqq n+1}\left|f\left(x_{j}\right)-q\left(x_{j}\right)\right| .
$$

It is known [1] that either $f\left(x_{j}\right)-\not{p}\left(x_{j}\right)=(-1)^{j} \rho(j=0,1, \cdots, n+1)$, or $f\left(x_{j}\right)-\tilde{p}\left(x_{j}\right)=-(-1)^{j} \rho(j=0,1, \cdots, n+1)$. In the first case let $u_{j}=$ $(-1)^{j}\left[f\left(x_{j}\right)-q\left(x_{j}\right)\right] / \rho^{\prime}(j=0,1, \cdots, n+1)$, and in the second case let $u_{j}=-(-1)^{j}\left[f\left(x_{j}\right)-q\left(x_{j}\right)\right] / \rho^{\prime}(j=0,1, \cdots, n+1)$. Then [1]

$$
\begin{array}{r}
1-u_{j} \leqq\left[\epsilon \sum_{\nu=0}^{n+1} A_{\nu}\right] /\left[(1+\epsilon) A_{j}\right] \text { where } A_{j}=\prod_{\substack{0 \leqq \alpha<\beta \leqq n+1 ; \alpha \neq j, \beta \neq j \\
(j=0,1, \cdots, n+1) .}}\left(x_{\beta}-x_{\alpha}\right) \\
\quad .
\end{array}
$$

From (8) one easily deduces that

$$
A_{j}>\left[\prod_{\nu=1}^{n} \nu !\right]\left(\begin{array}{c}
n+1 \\
j
\end{array}\right)\left[2 \rho_{1}(n+1) ! / M\right]^{\left(\begin{array}{c}
n+1 \\
2
\end{array}\right)} \quad(j=0,1, \cdots, n+1) .
$$

Also [4]

$$
A_{j} \leqq\left(\frac{1}{2}\right)^{\left(\begin{array}{c}
n+1 \\
2
\end{array}\right)}\left[\left\{\prod_{\nu=2}^{n+1} \nu^{\nu}\right\}\left\{\prod_{\nu=0}^{n-1} \nu^{\nu}\right\}\left\{\prod_{\nu=1}^{n}(2 \nu-1)^{2 \nu-1}\right\}^{-1}\right]^{1 / 2}
$$

Consequently, for every $j,\left(\sum_{\nu=0}^{n+1} A_{\nu}\right) / A_{j}<U$ and therefore, by (9),

$$
1-u_{j}<\epsilon U /(1+\epsilon) \text {. }
$$

For $j=0,1, \cdots, n+1$,

$$
\begin{aligned}
\left|\tilde{p}\left(x_{j}\right)-q\left(x_{j}\right)\right| & =\left|\left\{f\left(x_{j}\right)-q\left(x_{j}\right)\right\}-\left\{f\left(x_{j}\right)-\not{p}\left(x_{j}\right)\right\}\right| \\
& =\left|u_{j} \rho^{\prime}-\rho\right|=\left|u_{j}\left(\rho^{\prime}-\rho\right)-\rho\left(1-u_{j}\right)\right| \\
& \leqq \rho^{\prime}-\rho+\rho\left(1-u_{j}\right)<\epsilon \rho[1+\{U /(1+\epsilon)\}] \\
& \leqq \epsilon V[1+\{U /(1+\epsilon)\}] \leqq \eta / c .
\end{aligned}
$$

By Lagrange's interpolation formula and by the Lemma, for every $x \in[0,1]$,

$$
\begin{aligned}
& |\tilde{p}(x)-q(x)| \\
& \quad \leqq \sum_{j=0}^{n}\left|\tilde{p}\left(x_{j}\right)-q\left(x_{j}\right)\right| \prod_{\nu=0, \nu \neq j}^{n}\left|x-x_{\nu}\right| /\left|x_{j}-x_{\nu}\right| \\
& \quad<\sum_{j=0}^{n}(\eta / c)\left[\prod_{\nu=0}^{n-1} 1-\left\{2 \rho_{1} \nu(n+1) ! / M\right\}\right] /\left[j !(n-j) !\left\{2 \rho_{1}(n+1) ! / M\right\}^{n}\right]=\eta .
\end{aligned}
$$


7. TheOREm 2. Let $n(\geqq 1)$ be an integer, $f$ a real function satisfying throughout $[0,1]$, for some constant $\lambda$,

$$
\left|f\left(x_{2}\right)-f\left(x_{1}\right)\right| \leqq \lambda\left|x_{2}-x_{1}\right| .
$$

Let $S_{n+2}, \rho_{1}, \mu, V$ have the same meaning as in Theorem 1 and assume $\rho_{1}>0$. Let $\theta$ be an arbitrary number with $0<\theta<1$. Let $R$ be such that for some polynomial $Q(x)$ with real coefficients we have, throughout $[0,1],|f(x)-Q(x)| \leqq \theta \rho_{1},\left|Q^{n+1}(x)\right| \leqq R$. (Explicit values for such an $R$, depending on $\lambda, \rho_{1}, \mu$ and $\theta$ only, are given in the proof, where we determine also a desirable choice for $\theta$.) Let

$$
\begin{aligned}
c=\left[R /\left\{\rho_{1}(1-\theta)(n+1) !\right\}\right]^{n}(n !)^{-1} \prod_{\nu=0}^{n-1} 1-\left\{2 \rho_{1} \nu(1-\theta)(n+1) ! / R\right\} \\
U=(n+2)\left[\prod_{\nu=1}^{n} \nu !\right]^{-1}\left[R /\left\{4 \rho_{1}(1-\theta)(n+1) !\right\}\right]^{\left(\begin{array}{c}
n+1 \\
2
\end{array}\right)} \\
\cdot\left[\left\{\prod_{\nu=2}^{n+1} \nu^{\nu}\right\}\left\{\prod_{\nu=0}^{n-1} \nu^{\nu}\right\}\left\{\prod_{\nu=1}^{n}(2 \nu-1)^{2 \nu-1}\right\}^{-1}\right]^{1 / 2} .
\end{aligned}
$$

$V$ and $R$ are positive since $\rho_{1}>0$. Also, $c$ is positive as shown below. Let $\eta$ be an arbitrary positive number. Let $\epsilon$ and $c_{0}, c_{1}, \cdots, c_{n}$ be defined as in Theorem 1 , and let $C$ be an arbitrary finite subset of $[0,1]$ containing $\left\{c_{0}, \cdots, c_{n}\right\}$ and such that the maximal distance $d$ between two consecutive points of $C$ is smaller or equal to $\epsilon \rho_{1} /\left(\lambda+4 \mu n^{2}\right)$. Let $\rho, \tilde{p}, q$ be defined as in Theorem 1. Then again $\max _{0 \leqq x \leqq 1}|\tilde{p}(x)-q(x)|<\eta$.

Proof. Let $x_{0}, x_{1}, \cdots, x_{n+1}$ be as in the first sentence of the last proof. Let

$$
\rho_{0}=\min _{p \in P_{n}} \max _{0 \leqq j \leqq n+1}\left|Q\left(x_{j}\right)-p\left(x_{j}\right)\right|=\max _{0 \leqq j \leqq n+1}\left|Q\left(x_{j}\right)-p^{*}\left(x_{j}\right)\right| \quad\left(p^{*} \in P_{n}\right) .
$$

Let $x_{h}$ be such that $\max _{0 \leqq j \leqq n+1}\left|f\left(x_{j}\right)-p^{*}\left(x_{j}\right)\right|=\left|f\left(x_{h}\right)-p^{*}\left(x_{h}\right)\right|$. We have [1] $\rho_{0} \geqq\left|Q\left(x_{h}\right)-p^{*}\left(x_{h}\right)\right| \geqq\left|f\left(x_{h}\right)-p^{*}\left(x_{h}\right)\right|-\left|f\left(x_{h}\right)-Q\left(x_{h}\right)\right| \geqq \rho(1-\theta)$, and, by the theorem used to derive $(8)$, we have

$$
\begin{aligned}
& x_{\nu}-x_{\nu-1}>2 \rho_{0}(n+1) ! / \max _{0 \leqq x \leqq 1}\left|Q^{(n+1)}(x)\right| \\
& \geqq 2 \rho_{1}(1-\theta)(n+1) ! / R \quad(\nu=1,2, \cdots, n+1) .
\end{aligned}
$$

So $c>0$.

We shall now give explicitly two numbers, either of which can serve as an $R$. We start by mentioning the following result of Favard [5] and Ahiezer and Krein [6] which strengthens a previous result of D. Jackson. Let $F$ (with period $2 \pi$ ) map the reals into the reals and satisfy for every real $x_{1}, x_{2},\left|F\left(x_{2}\right)-F\left(x_{1}\right)\right| \leqq L\left|x_{2}-x_{1}\right|$, $L$ being a constant. Then for $N=0,1,2, \cdots$, there exists a trigonometric polynomial $T_{N}(x) \equiv \sum_{\nu=0}^{N} a_{\nu}{ }^{(N)} \cos (\nu x)+b_{\nu}{ }^{(N)} \sin (\nu x)$ such that

$$
\max _{0 \leqq x \leqq 2 \pi}\left|F(x)-T_{N}(x)\right| \leqq L \pi /\{2(N+1)\} .
$$

From this result one obtains by the method of Jackson [7] that for $N=0,1,2, \cdots$ there exists a $p_{N}(x) \equiv \sum_{\nu=0}^{N} c_{\nu}{ }^{(N)} x^{\nu}, c_{\nu}{ }^{(N)}$ being reals, such that

$$
\max _{0 \leqq x}\left|f(x)-p_{N}(x)\right| \leqq \lambda \pi /\{4(N+1)\}
$$


Let $m$ be the smallest integer $N(\geqq 0)$ such that $\lambda \pi /\{4(N+1)\} \leqq \theta \rho_{1}$, and let $Q(x) \equiv p_{m}(x)$. Then throughout $[0,1]$ we have $|f(x)-Q(x)| \leqq \theta \rho_{1},|Q(x)|$ $\leqq \mu+\theta \rho_{1}$. Therefore, by a theorem of W. Markoff [8],

$$
\begin{aligned}
\max _{0 \leqq x \leqq 1}\left|Q^{(n+1)}(x)\right| & \leqq 2^{n+1}\left(\mu+\theta \rho_{1}\right) \prod_{\nu=0}^{n}\left(m^{2}-\nu^{2}\right) /(2 \nu+1) \\
& <2^{n+1}\left(\mu+\theta \rho_{1}\right)\left[\lambda \pi /\left(4 \theta \rho_{1}\right)\right]^{2 n+2} / \prod_{\nu=0}^{n}(2 \nu+1)
\end{aligned}
$$

which gives two values for an $R$.

One can proceed now as in the proof of Theorem 1 (from the first sentence following (8) on), and conclude that $\max _{0 \leqq x \leqq 1}|\tilde{p}(x)-q(x)|<\eta$.

We finally make the following remark on the choice of $\theta$. One naturally seeks to take $d$ (and therefore $\epsilon$ ) as large as possible. If we take $\epsilon=\eta\{c V(1+U)\}^{-1}$, then we are interested in minimizing $U$, i.e., minimizing $R /(1-\theta)$. Suppose we take $R=2^{n+1}\left(\mu+\theta \rho_{1}\right)\left[\lambda \pi /\left(4 \theta \rho_{1}\right)\right]^{2 n+2} / \prod_{\nu=0}^{n}(2 \nu+1)$. Then we want to minimize $\left(\mu+\theta \rho_{1}\right) \theta^{-2 n-2} /(1-\theta)$, and as one easily sees, we have to choose $\theta$ for this purpose as the positive root of $\rho_{1} x^{2}+\left[(2 n+3) \mu-(2 n+1) \rho_{1}\right][2(n+1)]^{-1} x-\mu=0$.

Aerospace Research Laboratories

Wright-Patterson Air Force Base, Ohio

1. Ch. de la Vallege Poussin, Leçons sur l'Approximation des Fonctions d'une Variable Réelle, Paris, 1919, Chapter VI.

2. R. J. Duffin \& A. C. Schaeffer, Trans. Amer. Math. Soc., v. 50, 1941, pp. 517-528.

3. A. Markoff, Bull. Acad. Sci. St. Petersburg, v. 62, 1889, pp. 1-24.

4. I. SCHUR, Math. Z., v. 1, 1918, pp. 377-402.

5. J. FAvARD, Bull. Sci. Math., v. 61, 1937, pp. 209-224, 243-256.

6. N. Ahiezer \& M. KReIN, Dokl. Akad. Nauk SSSR, v. 15, 1937, pp. 107-112.

7. D. JACKson, The Theory of Approximation, Amer. Math. Soc., Providence, R. I., 1930, pp. 13-14.

8. W. MarkofF' (German translation), Math. Ann., v. 77, 1916, pp. 213-258. 